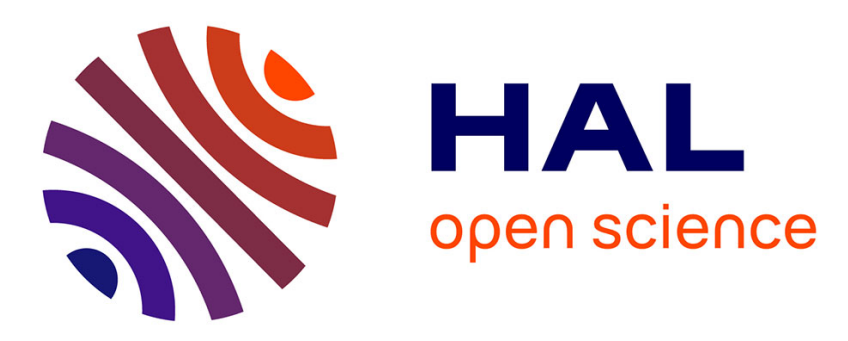

\title{
Spatial correlation characterization of a uniform circular array in 3D MIMO systems
}

Qurrat-Ul-Ain Nadeem, Abla Kammoun, Merouane Debbah, Mohamed-Slim

Alouini

\section{- To cite this version:}

Qurrat-Ul-Ain Nadeem, Abla Kammoun, Merouane Debbah, Mohamed-Slim Alouini. Spatial correlation characterization of a uniform circular array in 3D MIMO systems. 17th International Workshop on Signal Processing Advances in Wireless Communications (SPAWC), Jul 2016, Edinburgh, United Kingdom. 10.1109/SPAWC.2016.7536796 . hal-01789307

\section{HAL Id: hal-01789307 \\ https://hal-centralesupelec.archives-ouvertes.fr/hal-01789307}

Submitted on 25 Jul 2018

HAL is a multi-disciplinary open access archive for the deposit and dissemination of scientific research documents, whether they are published or not. The documents may come from teaching and research institutions in France or abroad, or from public or private research centers.
L'archive ouverte pluridisciplinaire HAL, est destinée au dépôt et à la diffusion de documents scientifiques de niveau recherche, publiés ou non, émanant des établissements d'enseignement et de recherche français ou étrangers, des laboratoires publics ou privés. 


\title{
Spatial Correlation Characterization of a Uniform Circular Array in 3D MIMO Systems
}

\author{
Qurrat-Ul-Ain Nadeem*, Abla Kammoun*, Mérouane Debbah ${ }^{\dagger}$, and Mohamed-Slim Alouini* \\ * CEMSE Division, King Abdullah University of Science and Technology (KAUST), Thuwal, Saudi Arabia. \\ Email: \{qurratulain.nadeem,abla.kammoun,slim.alouini\}@ kaust.edu.sa \\ $\dagger$ Mathematical and Algorithmic Sciences Lab, Huawei France R\&D, Paris, France \\ and CentraleSupélec, Gif-sur-Yvette, France. Email: merouane.debbah@ huawei.com
}

\begin{abstract}
In this paper, we consider a uniform circular array (UCA) of directional antennas at the base station (BS) and the mobile station (MS) and derive an exact closed-form expression for the spatial correlation present in the 3D multiple-input multiple-output (MIMO) channel constituted by these arrays. The underlying method leverages the mathematical convenience of the spherical harmonic expansion (SHE) of plane waves and the trigonometric expansion of Legendre polynomials. In contrast to the existing results, this generalized closed-form expression is independent of the form of the underlying angular distributions and antenna patterns. Moreover, the incorporation of the elevation dimension into the antenna pattern and channel model renders the proposed expression extremely useful for the performance evaluation of 3D MIMO systems in the future. The simulation results not only verify the derived analytical expression but also highlight the dependence of the spatial correlation on channel and array parameters. An interesting interplay between the mean angle of departure $(\mathrm{AoD})$, angular spread and the positioning of antennas in the array is demonstrated.
\end{abstract}

\section{INTRODUCTION}

Antenna arrays have become increasingly popular in the recent years to enhance signal quality, coverage and capacity. The performance of these arrays is governed by the spatial properties of the multiple-input multiple-output (MIMO) channels constituted by them. An important such statistical property is the spatial correlation. The spatial constraints at the base station (BS) and the mobile station (MS) limit the achievable user throughput by increasing the correlation in contemporary cellular networks. Consequently, it is imperative to characterize this correlation and study its impact on the system performance.

MIMO systems were initially designed to support antenna configurations capable of adaptation in the azimuth only. Consequently, most of the prior works on spatial correlation considered 2D cellular layouts and investigated the correlation in the azimuth only [1]-[3]. However, because of the 3D nature of real world transmission channels, beamforming in the azimuth alone can not fully exploit all the degrees of freedom offered by the channel. With the advent of 3D directional smart antennas, the elevation plane of the antenna pattern can also be used for performance optimization [4], [5].

An efficient approach to maximize the system capacity while meeting the spatial constraints is to pack these directional antennas intelligently such that the resulting antenna configuration minimizes the overall correlation. This has led to the emergence of several compact antenna array topologies. One such candidate antenna topology is the uniform circular array (UCA). The existing correlation models developed for the UCA topology are derived for a particular distribution of Angle of Departure (AoD) and Angle of Arrival (AoA) [1]-[3], [6]. The authors in [1] derived the spatial correlation expressions for Gaussian and uniform angular distributions. The proposed method could not admit a closed-form solution for the Gaussian angular distribution and relied on numerical integration methods to compute the correlation coefficients. Similarly, the spatial correlation could only be evaluated numerically for a cosine-shaped angular distribution in [3]. Some closed-form expressions were derived in [2] for the Laplacian azimuth AoA distribution, by making small angular spread approximations. Such assumptions might lead to useful expressions but do not accurately represent the characteristics of realistic propagation environments.

In this paper, we derive a general closed-form expression for the spatial correlation for the UCA topology in a 3D cellular layout by leveraging the mathematical convenience of the spherical harmonic expansion (SHE) of plane waves and the trigonometric expansion of Legendre polynomials. The final closed-form expression depends on the channel and array parameters through the Fourier Series (FS) coefficients of power azimuth and elevation spectrums. The novelty of the proposed method lies in the fact that it is valid for any 3D propagation environment. The analysis follows the guidelines in [7], where the spatial correlation in a uniform linear array (ULA) of antennas was investigated. The derivation for the UCA is not only more involved due to the intricate nature of the array response vector but also important owing to the several advantages the UCA offers over the ULA topology [8]. Numerical results corroborate the proposed expression and provide some useful insights into the impact of different channel conditions on the spatial correlation. The dependency of the correlation on array parameters like the relative position of antennas and the angular parameters parametrized by the mean and the spread is investigated.

The rest of the paper is organized as follows. Section II describes the 3D channel model. The closed-form expression for the spatial correlation is derived in section III. Section IV provides numerical results and section $\mathrm{V}$ concludes the paper.

\section{Channel Model and Antenna Configuration}

The 3D MIMO channel model used in this work is based on the latest 3GPP standards and WINNER+ [9], [10], which 
follow a geometry-based stochastic channel modeling approach. Channel realizations are generated by summing contributions of $N$ multiple paths with channel parameters like delay, power, AoD and AoA including both azimuth and elevation. In this section, we discuss the antenna configuration under investigation and present the corresponding 3D channel model.

In the UCA configuration, antenna ports are arranged in a circle of radius $r$ in the $\left(\hat{\mathbf{e}}_{x}, \hat{\mathbf{e}}_{y}\right)$ plane with the reference point at the center of the circle. The configuration is shown in Fig. 1. The 3GPP standards and ITU approximate the main lobe of the antenna pattern of each port by a narrow beam in the elevation [11], [12]. The horizontal and vertical antenna patterns at the transmitter can be approximated in dB as [7], [12],

$$
\begin{aligned}
g_{t, H}(\phi) & =-12\left(\frac{\phi}{\phi_{3 d B}}\right)^{2} \mathrm{~dB}, \\
g_{t, V}\left(\theta, \theta_{t i l t}\right) & =-12\left(\frac{\theta-\theta_{\text {tilt }}}{\theta_{3 d B}}\right)^{2} \mathrm{~dB},
\end{aligned}
$$

where $\phi_{3 d B}$ and $\theta_{3 d B}$ are the horizontal and vertical $3 \mathrm{~dB}$ beamwidths respectively. The individual antenna radiation pattern at the MS, $g_{r}(\varphi, \vartheta)$, is taken to be $0 \mathrm{~dB}$ since the MS should generally not favor any direction.

Based on the 3GPP activity around 3D channel modeling, the effective channel between the BS antenna port $s$ and the MS antenna port $u$ is given by [9], [13],

$$
\begin{aligned}
{[\mathbf{H}]_{s u} } & =\sum_{n=1}^{N} \alpha_{n} \sqrt{g_{t}\left(\phi_{n}, \theta_{n}, \theta_{t i l t}\right)} \sqrt{g_{r}\left(\varphi_{n}, \vartheta_{n}\right)}\left[\mathbf{a}_{r}\left(\varphi_{n}, \vartheta_{n}\right)\right]_{u} \\
& \times\left[\mathbf{a}_{t}\left(\phi_{n}, \theta_{n}\right)\right]_{s},
\end{aligned}
$$

where $\alpha_{n}$ is the complex amplitude of the $n^{\text {th }}$ path, assumed to be an i.i.d $\mathcal{N}\left(0, \frac{1}{N}\right)$ random variable (RV), $\theta_{\text {tilt }}$ is the downtilt angle, $\left(\phi_{n}, \theta_{n}\right)$ are the azimuth and elevation $\operatorname{AoDs}$ and $\left(\varphi_{n}, \vartheta_{n}\right)$ are the azimuth and elevation AoAs of the $n^{t h}$ path respectively. Note that $g_{t}\left(\phi_{n}, \theta_{n}, \theta_{t i l t}\right) \approx g_{t, H}\left(\phi_{n}\right) g_{t, V}\left(\theta_{n}, \theta_{\text {tilt }}\right)$, where $g_{t, H}(\phi)$ and $g_{t, V}\left(\theta, \theta_{t i l t}\right)$ are given by equations (1) and (2) respectively. Finally, vectors $\mathbf{a}_{t}(\phi, \theta)$ and $\mathbf{a}_{r}(\varphi, \vartheta)$ capture the antenna array responses. Their entries are given by,

$$
\begin{aligned}
{\left[\mathbf{a}_{t}(\phi, \theta)\right]_{s} } & =\exp \left(i \mathbf{k}_{t} \cdot \mathbf{x}_{s}\right), \\
{\left[\mathbf{a}_{r}(\varphi, \vartheta)\right]_{u} } & =\exp \left(i \mathbf{k}_{r} \cdot \mathbf{x}_{u}\right),
\end{aligned}
$$

where $\mathbf{x}_{s}$ is the location vector of the $s^{\text {th }}$ transmit (Tx) antenna, $\mathbf{x}_{u}$ is the location vector of the $u^{t h}$ receive (Rx) antenna, . is the scalar product, $\mathbf{k}_{t}$ and $\mathbf{k}_{r}$ are the Tx and Rx wave vectors, where $\mathbf{k}=k \hat{\mathbf{v}}$, with $k=\frac{2 \pi}{\lambda}, \lambda$ being the carrier wavelength and $\hat{\mathbf{v}}$ being the direction of wave propagation.

Given the UCA configuration in Fig. 1, $\hat{\mathbf{v}}_{t} \cdot \hat{\mathbf{x}}_{s}=\cos (\phi-$ $\left.\psi_{s}\right) \sin \theta_{n}$, where $\psi_{s}=\frac{2 \pi s}{N_{B S}}, s=0, \ldots N_{B S}-1$ and $\hat{\mathbf{v}}_{r} \cdot \hat{\mathbf{x}}_{u}=$ $\cos \left(\varphi-v_{u}\right) \sin \vartheta_{n}$, where $v_{u}=\frac{2 \pi u}{N_{M S}}, u=0, \ldots N_{M S}-1$. The effective channel between the $s^{t h}$ Tx antenna port and the $u^{t h}$ MS antenna in (3) can therefore be written as,

$$
\begin{aligned}
& {[\mathbf{H}]_{s u}=\sum_{n=1}^{N} \alpha_{n} \sqrt{g_{t}\left(\phi_{n}, \theta_{n}, \theta_{t i l t}\right)} \exp \left(i \frac{2 \pi}{\lambda} r \cos \left(\phi_{n}-\psi_{s}\right) \sin \theta_{n}\right)} \\
& \sqrt{g_{r}\left(\varphi_{n}, \vartheta_{n}\right)} \exp \left(i \frac{2 \pi}{\lambda} r \cos \left(\varphi_{n}-v_{u}\right) \sin \vartheta_{n}\right) .
\end{aligned}
$$

\section{Spatial Correlation based on the Fourier COEFFICIENTS OF POWER SPECTRUMS}

In this section, a general analytic expression for the spatial correlation between the Tx antenna ports is derived for the UCA configuration, considering realistic antenna patterns and arbitrary AoD and AoA distributions. The novelty of the proposed method lies in the fact that it is valid for any 3D propagation environment. We start this section by presenting the necessary tools and definitions required for the derivation.

\section{A. Power Spectrums}

The spatial correlation will be shown to have a direct dependence on the power azimuth spectrum (PAS) and the power elevation spectrum (PES), which describe the spatial distribution of the power in the azimuth and the elevation respectively. They are defined at the transmitter as,

$$
\begin{aligned}
\operatorname{PAS}_{t}(\phi) & =g_{t, H}(\phi) p_{\phi}(\phi), \\
\operatorname{PES}_{t}\left(\theta, \theta_{t i l t}\right) & =g_{t, V}\left(\theta, \theta_{t i l t}\right) p_{\theta}(\theta),
\end{aligned}
$$

where the azimuth angular power density function $p_{\phi}(\phi)=f_{\phi}(\phi)$, with $f_{\phi}(\phi)$ being the probability density function (pdf) of the azimuth angle. Similarly, the elevation angular power density function, $p_{\theta}(\theta)=\frac{f_{\theta}(\theta)}{\sin (\theta)}$, with $f_{\theta}(\theta)$ being the pdf of the elevation angle.

\section{B. Spherical Harmonics}

Spherical harmonics, $Y_{n}^{m}(\phi, \theta)$, provide orthonormal basis functions for the representation of functions on the 2-sphere (defined by $\mathbb{S}^{2} \triangleq\left\{\mathbf{x} \in \mathbb{R}^{3}:|x|=1\right\}$ ) and are defined for integer degree $n \geq 0$ and integer order $|m| \leq n$ as,

$$
Y_{n}^{m}(\hat{\mathbf{x}})=Y_{n}^{m}(\phi, \theta)=N_{n}^{m} P_{n}^{m}(\cos \theta) \exp (i m \phi),
$$

where $N_{n}^{m}=\sqrt{\frac{2 n+1(n-m) !}{4 \pi(n+m) !}}$ is the normalization constant .

In a $3 \mathrm{D}$ propagation environment, the antenna array response can be expanded using the spherical harmonic expansion (SHE) of plane waves given in the Jacobi-Anger result as [14],

$$
e^{i k \hat{\mathbf{v}} . \mathbf{x}}=\sum_{n=0}^{\infty} i^{n}(2 n+1) j_{n}(k\|\mathbf{x}\|) P_{n}(\hat{\mathbf{v}} . \hat{\mathbf{x}}), \mathbf{x} \in \mathbb{R}^{3},
$$

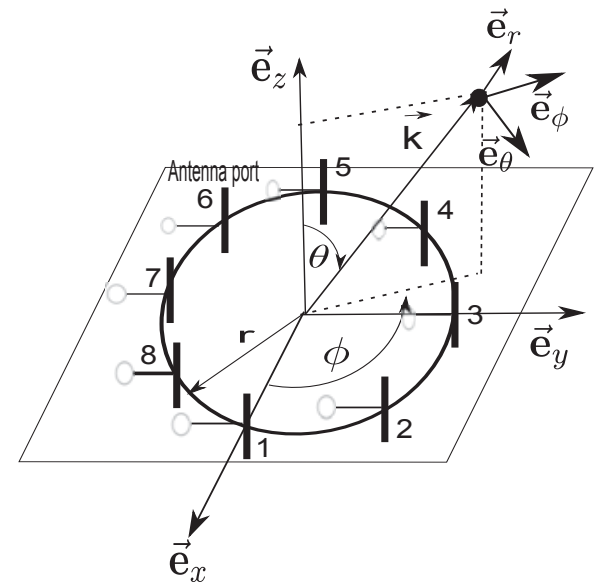

Fig. 1. Antenna configuration. 
where $j_{n}$ is the spherical Bessel function of order $n$ and $P_{n}$ is the Legendre polynomial function of order $n$. Let $\left(\phi_{1}, \theta_{1}\right)$ and $\left(\phi_{2}, \theta_{2}\right)$ be the spherical coordinates of vectors $\hat{\mathbf{v}}$ and $\mathbf{x}$ respectively, then by the Legendre addition theorem [15],

$$
\begin{aligned}
& P_{n}(\hat{\mathbf{v}} \cdot \hat{\mathbf{x}})=\frac{4 \pi}{2 n+1} \sum_{m=-n}^{m=n} Y_{n}^{m}(\hat{\mathbf{v}}) Y_{n}^{m *}(\hat{\mathbf{x}}) \\
& =P_{n}\left(\cos \theta_{1}\right) P_{n}\left(\cos \theta_{2}\right)+2 \sum_{m=1}^{n} \frac{(n-m) !}{(n+m) !} P_{n}^{m}\left(\cos \theta_{1}\right) \\
& \times P_{n}^{m}\left(\cos \theta_{2}\right) \cos \left[m\left(\phi_{1}-\phi_{2}\right)\right]
\end{aligned}
$$

where $P_{n}^{m}$ are the associated Legendre polynomials.

\section{Closed-form Expression for the Spatial Correlation}

The parameters describing the propagation paths in (6) are i.i.d, which allows us to simplify the double sum in the expression of the spatial correlation, $\rho=\mathbb{E}\left[\mathbf{H}_{s u} \mathbf{H}_{s^{\prime} u^{\prime}}^{H}\right]$ as,

$$
\begin{aligned}
& \rho=\mathbb{E}\left[\mathbf{H}_{s u} \mathbf{H}_{s^{\prime} u^{\prime}}^{H}\right]=\rho_{t}\left(s, s^{\prime}\right) \rho_{r}\left(u, u^{\prime}\right), \text { where } \\
& \rho_{t}\left(s, s^{\prime}\right)=\mathbb{E}\left[g _ { t } ( \phi , \theta , \theta _ { t i l t } ) \operatorname { e x p } \left(i \frac { 2 \pi } { \lambda } r \operatorname { s i n } \theta \left(\cos \left(\phi-\psi_{s}\right)\right.\right.\right. \\
& \left.\left.\left.-\cos \left(\phi-\psi_{s^{\prime}}\right)\right)\right)\right] \\
& \rho_{r}\left(u, u^{\prime}\right)=\mathbb{E}\left[g _ { r } ( \varphi , \vartheta ) \operatorname { e x p } \left(i \frac { 2 \pi } { \lambda } r \operatorname { s i n } \vartheta \left(\cos \left(\varphi-v_{u}\right)\right.\right.\right. \\
& \left.\left.\left.-\cos \left(\varphi-v_{u^{\prime}}\right)\right)\right)\right]
\end{aligned}
$$

where $\psi_{s}=\frac{2 \pi s}{N_{B S}}, \psi_{s^{\prime}}=\frac{2 \pi s^{\prime}}{N_{B S}}$ and $v_{u}=\frac{2 \pi u}{N_{M S}}, v_{u^{\prime}}=\frac{2 \pi u^{\prime}}{N_{M S}}$.

We now derive a general analytical expression for (13). The analysis for the UCA is harder than that for the ULA topology because (13) and (14) do not correspond directly to the SHE expression and need to be reformulated. We start our analysis by reformulating the expression in (13) in a way that the SHE result in (10) can be directly employed. Defining $Z_{1}=\cos \psi_{s}-\cos \psi_{s^{\prime}}$ and $Z_{2}=\sin \psi_{s}-\sin \psi_{s^{\prime}}, \rho_{t}\left(s, s^{\prime}\right)$ can be written as,

$\rho_{t}\left(s, s^{\prime}\right)=\mathbb{E}\left[g_{t}\left(\phi, \theta, \theta_{t i l t}\right) \exp \left(i \frac{2 \pi}{\lambda} r \sin \theta\left(Z_{1} \cos \phi+Z_{2} \sin \phi\right)\right)\right]$ $=\mathbb{E}\left[g_{t}\left(\phi, \theta, \theta_{t i l t}\right) \exp \left(\frac{i 2 \pi r}{\lambda} \sin \theta \sqrt{Z_{1}^{2}+Z_{2}^{2}} \cos \left(\phi-\tan ^{-1} \frac{Z_{2}}{Z_{1}}\right)\right.\right.$
Defining $\zeta=\tan ^{-1}\left(Z_{2} / Z_{1}\right)$ and $c=\sqrt{Z_{1}^{2}+Z_{2}^{2}}$, we have,

$$
\rho_{t}\left(s, s^{\prime}\right)=\mathbb{E}\left[g_{t}\left(\phi, \theta, \theta_{t i l t}\right) \exp \left(i \frac{2 \pi}{\lambda} r c \sin \theta \cos (\phi-\zeta)\right)\right]
$$

The spherical coordinates $\left(\phi_{1}, \theta_{1}\right)$ of the wave vector $k \hat{\mathbf{v}}$ are $(\phi, \theta)$, and the spherical coordinates $\left(\phi_{2}, \theta_{2}\right)$ of $\mathbf{x}$ i.e., the vector between the positions of $s^{t h}$ and $s^{\text {th }} \mathrm{Tx}$ antennas are $\left(\zeta, \frac{\pi}{2}\right)$. Combining the addition theorem in (11) with the SHE of the exponential term in (16) and using the resulting expression in (16) would expand $\rho_{t}\left(s, s^{\prime}\right)$ to yield (17).

Next we exploit the properties of Legendre and associated Legendre polynomials followed by some trigonometric manipulations to systematically expand (17) to yield (18), where $\bar{P}_{n}^{m}(\mathrm{x})=\sqrt{\left(n+\frac{1}{2}\right) \frac{(n-m) !}{(n+m) !}} P_{n}^{m}(\mathrm{x})$. The expansion looks involved at first sight but it will now be shown to yield an interesting closed-form expression that works for any arbitrary choice of antenna patterns and angular distributions. A sketch of the proof is provided here for readers' convenience.

It is not easy to derive a closed-form expression for the correlation, because the RVs, AoDs and AoAs, appear as arguments of Legendre polynomials in (18), making it difficult to compute the expectations with respect to these variables. However, the trigonometric expansion of Legendre polynomials given in [16] and stated below can be exploited to yield an interesting closed-form expression for $\rho_{t}\left(s, s^{\prime}\right)$.

$$
\begin{aligned}
P_{2 n}(\cos x) & =\sum_{k=-n}^{n} p_{n-k} p_{n+k} \cos (2 k x), \\
\bar{P}_{2 n}^{2 m}(\cos x) & =\sum_{k=0}^{n} c_{2 n, 2 k}^{2 m} \cos (2 k x), \\
\bar{P}_{2 n-1}^{2 m-1}(\cos x) & =\sum_{k=1}^{n} d_{2 n-1,2 k-1}^{2 m-1} \sin ((2 k-1) x),
\end{aligned}
$$

where $p_{n}, c_{2 n, 2 k}^{2 m}$ and $d_{2 n-1,2 k-1}^{2 m-1}$ are generated using recursion fellations in [16]. Using (19)-(21), the expectations in (18) can be expressed analytically as a linear combination of the FS

$$
\begin{aligned}
& \left.\rho_{t}\left(s, s^{\prime}\right)=\mathbb{E}\left[g_{t}\left(\phi, \theta, \theta_{t i l t}\right) \sum_{n=0}^{\infty} i^{n}(2 n+1) j_{n}\left(\frac{2 \pi}{\lambda} r c\right)\left(P_{n}(\cos \theta) P_{n}(0)+2 \sum_{m=1}^{n} \frac{(n-m) !}{(n+m) !} P_{n}^{m}(\cos \theta) P_{n}^{m}(0) \cos (m(\phi-\zeta))\right)\right](17)\right]\left(\frac{2 \pi}{\lambda} r c\right) P_{2 n}(0) \mathbb{E}\left[P_{2 n}(\cos \theta) g_{t, V}\left(\theta, \theta_{t i l t}\right)\right] \mathbb{E}\left[g_{t, H}(\phi)\right] \\
& \rho_{t}\left(s, s^{\prime}\right)=\mathbb{E}\left[g_{t}\left(\phi, \theta, \theta_{t i l t}\right)\right] j_{0}\left(\frac{2 \pi}{\lambda} r c\right)+\sum_{n=1}^{\infty}(-1)^{n}(4 n+1) j_{2 n}\left(\frac{2 \pi}{\lambda} r c\right)\left(\sum _ { m = 1 } ^ { n } \overline { P } _ { 2 n } ^ { 2 m } ( 0 ) \mathbb { E } [ \overline { P } _ { 2 n } ^ { 2 m } ( \operatorname { c o s } \theta ) g _ { t , V } ( \theta , \theta _ { t i l t } ) ] \left(\cos (2 m \zeta) \mathbb{E}\left[\cos (2 m \phi) g_{t, H}(\phi)\right]\right.\right. \\
& \quad+\sum_{n=1}^{\infty} 4(-1)^{n} j_{2 n}\left(\frac{2 \pi}{\lambda} r c\right)\left(\sum_{m=1}^{n} \bar{P}_{2 n-1}^{2 m-1}(0) \mathbb{E}\left[\bar{P}_{2 n-1}^{2 m-1}(\cos \theta) g_{t, V}\left(\theta, \theta_{t i l t}\right)\right]\right. \\
& \left.\left.\left.\left.\left.+\sin (2 m \zeta) \mathbb{E}\left[\sin (2 m \phi) g_{t, H}(\phi)\right]\right)\right)-\sum_{n=1}^{\infty} 4 i(-1)^{n} j_{2 n-1}\left(\frac{2 \pi}{\lambda}\right) g_{t, H}(\phi)\right]+\sin ((2 m-1) \zeta) \mathbb{E}\left[\sin ((2 m-1) \phi) g_{t, H}(\phi)\right]\right)\right) \\
& \quad \times(\cos ((2 m-1) \zeta) \mathbb{E}[\cos ((2 m-1) \phi)
\end{aligned}
$$


coefficients of PAS and PES, which are defined as,

$$
\begin{aligned}
a_{\phi}(m) & =\frac{1}{\pi} \int_{-\pi}^{\pi} \operatorname{PAS}_{t}(\phi) \cos (m \phi) d \phi, \\
b_{\phi}(m) & =\frac{1}{\pi} \int_{-\pi}^{\pi} \operatorname{PAS}_{t}(\phi) \sin (m \phi) d \phi, \\
a_{\theta}(k) & =\frac{1}{\pi} \int_{0}^{2 \pi} \operatorname{PES}_{t}\left(\theta, \theta_{t i l t}\right) \cos (k \theta) d \theta, \\
b_{\theta}(k) & =\frac{1}{\pi} \int_{0}^{2 \pi} \operatorname{PES}_{t}\left(\theta, \theta_{t i l t}\right) \sin (k \theta) d \theta .
\end{aligned}
$$

We expand one of the expectation terms in (18) as,

$$
\begin{aligned}
\mathbb{E} & {\left[P_{2 n}(\cos \theta) g_{t, V}\left(\theta, \theta_{t i l t}\right)\right]=\sum_{k=-n}^{n}\left[p_{n-k} p_{n+k} \int_{0}^{2 \pi} \cos (2 k \theta)\right.} \\
\times & \left.g_{t, V}\left(\theta, \theta_{t i l t}\right) p(\theta) \sin (\theta) d \theta\right], \\
& =\sum_{k=-n}^{n} p_{n-k} p_{n+k}\left[\frac{1}{2} \int_{0}^{2 \pi} \sin ((2 k+1) \theta) \mathrm{PES}_{t}\left(\theta, \theta_{t i l t}\right) d \theta\right. \\
& \left.-\frac{1}{2} \int_{0}^{2 \pi} \sin ((2 k-1) \theta) \mathrm{PES}_{t}\left(\theta, \theta_{t i l t}\right) d \theta\right], \\
& =\sum_{k=-n}^{n} p_{n-k} p_{n+k} \frac{\pi}{2}\left[b_{\theta}(2 k+1)-b_{\theta}(2 k-1)\right] .
\end{aligned}
$$

The same can be done for the other expectation terms. With the help of all these tools, we now present in (27) the generalized closed-form expression for the correlation between any pair of Tx antenna ports. An expression for $\rho_{r}\left(u, u^{\prime}\right)$ can be obtained similarly. Consequently, the correlation between any pair of channels constituted by distinct pairs of Tx antenna ports and Rx antenna ports is given by (12). Note that (27) involves an infinite summation over $n$, which can be truncated to a small finite number, $N_{0}$, of terms such that the truncation error has a bound that decreases exponentially with $N_{0}$ [7].

\section{Simulation Results}

In order to validate the proposed generalized spatial correlation expression, we simulate (27) using the angular distributions and antenna patterns specified in the standards. The elevation AoD and AoA are drawn from the Laplacian elevation density spectrum with parameters, $\left(\theta_{0}, \sigma\right)$ that denote the mean AoD/AoA

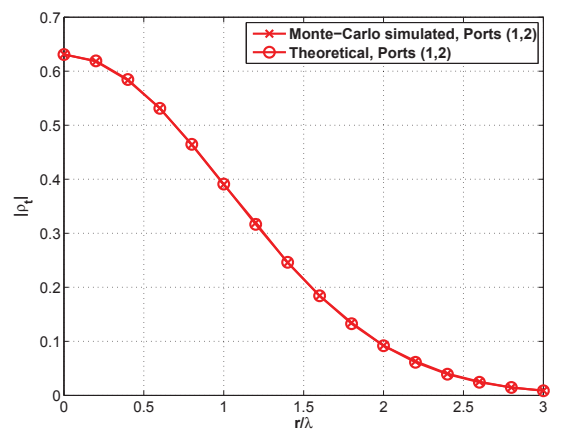

Fig. 2. Correlation between adjacent Tx antenna ports.

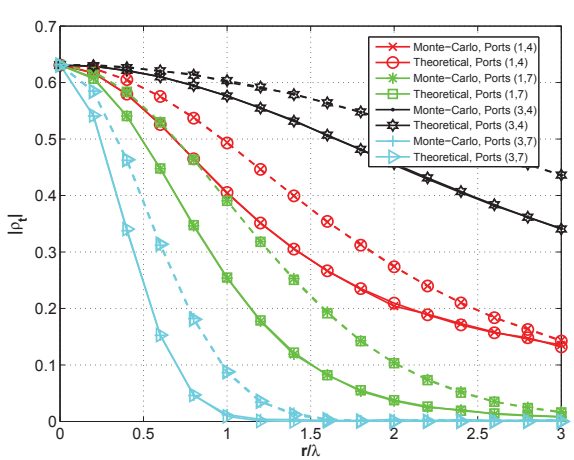

Fig. 3. Correlation between different pairs of $\mathrm{Tx}$ antenna ports for $\mu=0$. The solid lines correspond to $\kappa_{s}=20$ and the dotted lines correspond to $\kappa_{s}=40$.

and the angular spread in the elevation respectively. In the recent years, the Von Mises (VM) distribution has received great attention in modeling nonisotropic propagation. This distribution, with parameters $\left(\mu, \frac{1}{\kappa}\right)$ that denote the mean AoD/AoA and the measure of dispersion in the azimuth respectively, is used to generate the azimuth angles. Using these angular densities and the vertical antenna pattern in (2), the FS coefficients of PAS and PES are computed.

For the purpose of validation, we compute the correlation coefficients between antenna ports 1 and 2 in Fig. 1 using the theoretical result in (27) and the Monte-Carlo simulation of (13) over 10000 channel realizations. For the simulations, we set $N_{0}=20, \theta_{\text {tilt }}=95^{\circ}, \theta_{3 d B}=15^{\circ}, \phi_{3 d B}=70^{\circ}, \sigma_{s}=10^{\circ}$, $\theta_{0}=90^{\circ}, \kappa_{s}=20$ and $\mu=0$. The result is shown in Fig. 2 .

$$
\begin{aligned}
& \rho_{t}\left(s, s^{\prime}\right)=\pi^{2} a_{\phi}(0) b_{\theta}(1) j_{0}\left(\frac{2 \pi}{\lambda} r c\right)+\sum_{n=1}^{\infty}(-1)^{n}(4 n+1) j_{2 n}\left(\frac{2 \pi}{\lambda} r c\right) P_{2 n}(0) a_{\phi}(0) \pi^{2} \sum_{k=-n}^{n} p_{n-k} p_{n+k} \frac{1}{2}\left[b_{\theta}(2 k+1)\right. \\
& \left.-b_{\theta}(2 k-1)\right]+\sum_{n=1}^{\infty} 4(-1)^{n} j_{2 n}\left(\frac{2 \pi}{\lambda} r c\right)\left(\sum_{m=1}^{n} \bar{P}_{2 n}^{2 m}(0) \pi^{2}\left(\cos (2 m \zeta) a_{\phi}(2 m)+\sin (2 m \zeta) b_{\phi}(2 m)\right)\right. \\
& \left.\times \sum_{k=0}^{n} c_{2 n, 2 k}^{2 m} \frac{1}{2}\left[b_{\theta}(2 k+1)-b_{\theta}(2 k-1)\right]\right)-\sum_{n=1}^{\infty} 4 i(-1)^{n} j_{2 n-1}\left(\frac{2 \pi}{\lambda} r c\right)\left(\sum_{m=1}^{n} \bar{P}_{2 n-1}^{2 m-1}(0) \pi^{2}\right. \\
& \left.\times\left(\cos ((2 m-1) \zeta) a_{\phi}(2 m-1)+\sin ((2 m-1) \zeta) b_{\phi}(2 m-1)\right) \sum_{k=1}^{n} d_{2 n-1,2 k-1}^{2 m-1} \frac{1}{2}\left[a_{\theta}(2 k-2)-a_{\theta}(2 k)\right]\right)
\end{aligned}
$$




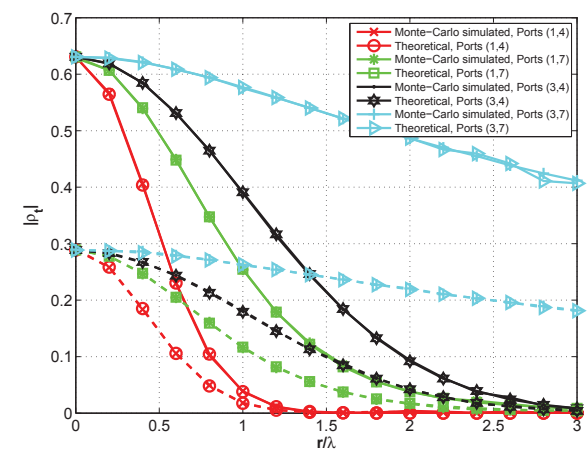

Fig. 4. Correlation between different pairs of ports for $\mu=\pi / 2$. The solid lines correspond to $\sigma_{s}=10^{\circ}$ and the dotted lines correspond to $\sigma_{s}=40^{\circ}$.

As expected, the correlation is seen to decrease as the radius of the array increases and more importantly, the theoretical result provides a perfect fit to the Monte-Carlo simulated correlation using less than 20 summations over $n$.

Next we study the correlation between different pairs of Tx antenna ports to see how this correlation varies with the position of the ports. The ports are numbered from 1 to 8 in order in Fig. 1. The validation of the theoretical result is done for different pairs of ports and is shown in Fig. 3. The values of the correlation are governed by the interplay between the relative positions of the ports and the values of the channel parameters like the mean AoD/AoA and the angular spread. The incorporation of antenna patterns also impacts the correlation. For example, out of $(1,4),(1,7),(3,4)$ and $(3,7)$ pairs of ports for which the result is shown, the correlation values between port 3 and port 4 are the highest since the relative distance between them is the shortest. The relative distance between the pair $(1,7)$ is shorter than that between $(1,4)$, but the correlation values for $(1,7)$ are lower than the values for the correlation between ports 1 and 4 . This is explained by observing that for the mean azimuth AoD $\mu=0^{\circ}$, the mean radiation is in $\hat{\mathbf{e}}_{x}$ direction and the spread is measured along $\hat{\mathbf{e}}_{y}$. The distance along $\hat{\mathbf{e}}_{y}$ is shorter for the pair $(1,4)$ yielding higher values of correlation for this pair as compared to the values for $(1,7)$. It is important to know that increasing $\kappa$ will cause the correlation values for all pairs to increase due to a decrease in the azimuthal spread. This can be seen by observing the dotted lines in Fig. 3 plotted for $\kappa_{s}=40$. Also, the effect of shorter inter-port distance will dominate with the decrease in spread.

Changing the value of $\mu$ to $\pi / 2$ yields a very different trend in the values of correlation as shown in Fig. 4 . Now between $(1,4)$ and $(1,7)$ pairs of antenna ports, the correlation in the pair $(1,7)$ is higher because of the shorter relative distance along the direction of the azimuthal spread, which is now measured along $\hat{\mathbf{e}}_{x}$. Also the pair $(3,7)$ has the highest correlation among the simulated cases because for $\mu=\pi / 2$, port 7 is directly behind port 3 and in line with the mean AoD. We also show in the same figure the effect of increasing $\sigma_{s}$ (see dotted lines) that causes the correlation to decrease. The value of $\rho_{t}$ at 0 antenna spacing is the average Tx power of the antenna which also decreases with the increase in spread due to the incorporation of the vertical antenna pattern into the channel model. Therefore the values of correlation are governed by both the channel and array parameters. The mean AoD/AoA and angular spread play a crucial role in governing the overall correlation in the channel.

\section{CONClusion}

We derived a closed-form expression for the spatial correlation in 3D MIMO channels with directional antennas arranged in a uniform circular fashion. Our approach leverages the mathematical convenience of the SHE of plane waves and the trigonometric expansion of Legendre and associated Legendre polynomials. The resulting expression is independent of the form of the underlying angular distribution and form of antenna pattern. Application of the result reveals a strong interplay between the array and channel parameters in determining the overall spatial correlation present in the channel.

\section{REFERENCES}

[1] J. Zhou, S. Sasaki, S. Muramatsu, H. Kikuchi, and Y. Onozato, "Spatial correlation for a circular antenna array and its applications in wireless communication," in IEEE Global Telecommunications Conference, GLOBECOM '03., vol. 2, Dec 2003, pp. 1108-1113.

[2] A. Forenza, D. J. Love, and R. W. Heath, "Simplified spatial correlation models for clustered MIMO channels with different array configurations," IEEE Transactions on Vehicular Technology, vol. 56, no. 4, pp. 1924-1934, July. 2007.

[3] J.-A. Tsai and B. Woerner, "The fading correlation function of a circular antenna array in mobile radio environment," in IEEE Global Telecommunications Conference, vol. 5, 2001, pp. 3232-3236.

[4] L. Thiele, T. Wirth, K. Brner, M. Olbrich, V. Jungnickel, J. Rumold, and S. Fritze, "Modeling of $3 \mathrm{~d}$ field patterns of downtilted antennas and their impact on cellular systems," in ITG Workshop Smart Antennas, 2009.

[5] A. Kuchar, J.-P. Rossi, and E. Bonek, "Directional macro-cell channel characterization from urban measurements," IEEE Transactions on Antennas and Propagation, vol. 48, no. 2, pp. 137-146, Feb. 2000.

[6] H. Rogier, "Spatial correlation in uniform circular arrays based on a spherical-waves model for mutual coupling," AEUE - International Journal of Electronics and Communications, vol. 60, no. 7, pp. 521-532, 2006.

[7] Q.-U.-A. Nadeem, A. Kammoun, M. Debbah, and M.-S. Alouini, "A generalized spatial correlation model for $3 \mathrm{~d}$ mimo channels based on the fourier coefficients of power spectrums," IEEE Transactions on Signal Processing,, vol. 63, no. 14, pp. 3671-3686, July 2015.

[8] J.-A. Tsai, R. Buehrer, and B. Woerner, "Ber performance of a uniform circular array versus a uniform linear array in a mobile radio environment," IEEE Transactions on Wireless Communications, vol. 3, no. 3, pp. 695-700, May 2004.

[9] 3GPP TR 36.873 V12.0.0 , "Study on 3D channel model for LTE," Sep. 2014.

[10] J. Meinilä, P. Kyösti et al., "D5.3: WINNER+ Final Channel Models V1.0," [Online.] Available: http://projects.celtic-initiative.org/winner+/WINNER+ \%20Deliverables/D5.3_v1.0.pdf, June. 2010.

[11] 3GPP TR 36.814 V9.0.0 , "Further advancements for E-UTRA physical layer aspects," March 2010.

[12] Report ITU-R M.2135, "Guidelines for evaluation of radio interface technologies for IMT-advanced," [Online]. Available: http://www.itu.int/ pub/R-REP-M.2135-2008/en, 2008.

[13] A. Kammoun, H. Khanfir, Z. Altman, M. Debbah, and M. Kamoun, "Preliminary results on 3D channel modeling: From theory to standardization," IEEE Journal on Selected Areas in Communications, vol. 32, no. 6, pp. 1219-1229, June. 2014.

[14] D. Colton and R. Kress, Inverse Acoustic and Electromagnetic Scattering Theory, 3rd ed. New York, NY: Springer, 2013.

[15] R. A. Kennedy and P. Sadeghi, Hilbert Space Methods in Signal Processing. Cambridge, UK: Cambridge University Press, 2013

[16] D. J. Hofsommer and M. L. Potters, "Table Of Fourier Coefficients of Assosciated Legendre Functions," R 478 Computation Department of the Mathematical Center, Amsterdam, June. 1960. 